\title{
BMJ Open China PEACE risk estimation tool for in-hospital death from acute myocardial infarction: an early risk classification tree for decisions about fibrinolytic therapy
}

\author{
Xi Li, ${ }^{1}$ Jing Li, ${ }^{1}$ Frederick A Masoudi, ${ }^{2}$ John A Spertus, ${ }^{3}$ Zhenqiu Lin, ${ }^{4}$ \\ Harlan M Krumholz, ${ }^{5}$ Lixin Jiang, ${ }^{1}$ for the China PEACE Collaborative Group
}

To cite: Li X, Li J, Masoudi FA, et al. China PEACE risk estimation tool for in-hospital death from acute myocardial infarction: an early risk classification tree for decisions about fibrinolytic therapy. BMJ Open 2016;6:e013355. doi:10.1136/bmjopen-2016013355

- Prepublication history and additional material is available. To view please visit the journal (http://dx.doi.org/ 10.1136/bmjopen-2016013355).

HMK and LJ are joint senior authors.

Received 7 July 2016 Accepted 27 September 2016

CrossMark

For numbered affiliations see end of article.

Correspondence to

Professor Lixin Jiang; jiangl@ fwoxford.org

\section{ABSTRACT}

Objectives: As the predominant approach to acute reperfusion for ST segment elevation myocardial infarction (STEMI) in many countries, fibrinolytic therapy provides a relative risk reduction for death of $\sim 16 \%$ across the range of baseline risk. For patients with low baseline mortality risk, fibrinolytic therapy may therefore provide little benefit, which may be offset by the risk of major bleeding. We aimed to construct a tool to determine if it is possible to identify a low-risk group among fibrinolytic therapyeligible patients.

Design: Cross-sectional study.

Setting: The China Patient-centered Evaluative Assessment of Cardiac Events (PEACE) study includes a nationally representative retrospective sample of patients admitted with acute myocardial infarction (AMI) in 162 hospitals.

Participants: 3741 patients with STEMI who were fibrinolytic-eligible but did not receive reperfusion therapy.

Main outcome measures: In-hospital mortality, which was defined as a composite of death occurring within hospitalisation or withdrawal from treatment due to a terminal status at discharge.

Results: In the study cohort, the in-hospital mortality was $14.7 \%$. In the derivation cohort and the validation cohort, the combination of systolic blood pressure ( $\geq 100 \mathrm{~mm} \mathrm{Hg}$ ), age ( $<60$ years old) and gender (male) identified one-fifth of the cohort with an average mortality rate of $<3.0 \%$. Half of this low risk group - those with non-anterior AMI-had an average in-hospital death risk of $1.5 \%$.

Conclusions: Nearly, one in five patients with STEMI who are eligible for fibrinolytic therapy are at a low risk for in-hospital death. Three simple factors available at the time of presentation can identify these individuals and support decision-making about the use of fibrinolytic therapy.

Trial registration number: NCT01624883.

\section{Strengths and limitations of this study}

- We identified a fibrinolytic-eligible but untreated cohort with similar risk profiles as their treated counterparts, in a nationally representative sample of patients with acute myocardial infarction.

- We used the classification and regression tree to gain a more nuanced view of interactions while maintaining a simple algorithm in identifying the low risk group.

- The decision analysis involved only the shortterm benefits; however, the entire benefit of fibrinolytic therapy was concentrated in shortterm period.

- Patient eligibility and data collection on some predictors were based on local diagnosis in medical records.

\section{INTRODUCTION}

Fibrinolytic therapy remains a mainstay of acute reperfusion for patients with ST segment elevation myocardial infarction (STEMI) in many countries and is the only treatment option in settings where primary percutaneous coronary intervention (PCI) is not available. ${ }^{1-3}$ Based on landmark trials, fibrinolytic therapy in eligible patients confers about a $16 \%$ relative risk reduction in short-term mortality. ${ }^{4}$ Clinical practice guidelines currently recommend the use of fibrinolytic therapy for all patients without contraindications who do not have timely access to primary PCI and present to the hospital within 12 hours of symptom onset, ${ }^{56}$ consider the treatment is reasonable for patients with clinical and/or ECG evidence of ongoing ischaemia within 12-24 hours of symptom onset. $^{78}$ 
The assumption of the guidelines is that all patients with STEMI deemed eligible by these general criteria should be treated. In landmark trials, subgroup analysis focused on the relative benefit in patients with different characteristics, but not the absolute reduction in mortality. ${ }^{4}$ However, for individual patients with a very low baseline risk of mortality without treatment, the absolute expected benefit of fibrinolytic therapy may be insufficient to justify the risks of major bleeding complications, including intracranial haemorrhage (ICH), which occurs in $0.5-1.0 \%$ of patients. ${ }^{4-12}$ Given the relative risk reduction, patients with baseline risks of $<3 \%$ would have an absolute mortality benefit roughly equivalent to that of ICH and may, thus, not merit treatment with fibrinolytic therapy. The question is whether such a low-risk group exists and can it be identified with information available at the time of presentation so as not to delay treatment for those who would benefit.

Estimating the baseline mortality risk of patients with STEMI requires study in a cohort of patients who did not receive reperfusion therapy. However, existing risk stratification tools, which typically include treated patients, are influenced by the impact of reperfusion therapy ${ }^{13-21}$ Identifying an untreated cohort in contemporary practice is challenging as many studies found that in most countries the majority of patients with STEMI receive a reperfusion, those untreated are often with bias in clinical and demographic characteristics. However, in China, about half of eligible patients with STEMI do not receive reperfusion therapy, mainly due to suboptimal system performance. ${ }^{22}$ Thus, there are large numbers of untreated eligible patients of similar profile with their treated counterpart, providing a rare opportunity to assess the baseline risk. The individual risk evaluation could be of significant value for China and other countries, where fibrinolytic therapy is commonly used to facilitate a more informed, patientcentred decision-making.

Accordingly, we sought to construct a risk tool from fibrinolytic-eligible, but untreated, patients hospitalised with STEMI in China. We developed a classification tree to estimate the baseline risk among eligible patients with the objective of identifying a population at such a low risk of death that the net benefits of fibrinolytic therapy for them may be marginal. The goal of this study, as part of a government-funded project to improve care for patients with acute myocardial infarction (AMI), was to equip clinicians with a simple risk tool that can be used at the time of presentation that can complement clinical judgement for this important individualised decision.

\section{METHODS}

\section{Data source}

The design of the China Patient-centered Evaluative Assessment of Cardiac Events (PEACE) Retrospective Study of AMI (China PEACE-Retrospective AMI Study) has been published previously. ${ }^{23}$ In brief, we used a stratified two-stage cluster random sampling design, including 162 hospitals in urban and rural regions in China. We obtained a nationally representative sample of discharges in 2001, 2006 and 2011, with confirmed diagnosis of AMI (International Classification of Diseases, Ninth Revision, Clinical Modification codes 410.xx, International Classification of Diseases, Tenth Revision, Clinical Modification codes I21.xx or text of AMI). We established a data set for medical records abstraction including demographic information, characteristics at presentation, laboratory parameters, in-hospital therapy, timing of care delivery and in-hospital clinical outcomes, with standard definitions. Using standardised central medical record abstraction, data quality was monitored using rigorous quality assurance measures, with overall accuracy of $>98 \%$ (see online supplementary appendix 1 ).

The Chinese government, which provided financial support, had no role in the design or conduct of the study; in the collection, management, analysis and interpretation of the data; or in the preparation or approval of the manuscript. The ethics committee of the China National Center for Cardiovascular Diseases approved the study. The study is registered at http://www. clinicaltrials.gov (NCT01624883).

\section{Study cohort}

To establish the risk tool, we identified fibrinolytic-eligible patients admitted in any hospital who did not receive reperfusion therapy during their hospitalisation. We excluded the patients who were transferred to other hospitals due to lack of treatment outcomes. We also excluded those who were discharged alive within 24 hours, because it is likely that they left against medical advice and there was very little time for follow-up. Eligibility was defined as patients with STEMI who arrived to the hospital within 24 hours of symptom onset, according to class I and IIa guideline recommendations, and did not have contraindications to fibrinolytic therapy, including history of haemorrhagic stroke, active bleeding at presentation, or any other physician-documented contraindication. ${ }^{7}$ The diagnosis of STEMI was determined by the combination of clinical discharge diagnosis terms and ECG results. If the local diagnosis was not definitive, cardiologists at the coordinating centre reviewed the medical record and ECG. We considered left bundle branch block (LBBB) as a STEMI equivalent. The type of AMI was validated by review of ECG from randomly selected records by a cardiologist not involved in abstraction (see online supplementary appendix 2).

\section{Definition of outcomes}

The outcome was in-hospital mortality, which was defined as a composite of death occurring within hospitalisation or withdrawal from treatment due to a terminal status at discharge. In China, it is common for patients who are clinically failing to request to withdraw treatment and be discharged home to die. According to 
follow-up reports by local physicians, $90 \%$ of the patients who withdrew from treatment due to a terminal status had died within 3 days after the discharge.

\section{Candidate predictors}

We reviewed prior studies and identified potential predictors of mortality in four categories: (1) demographic characteristics; (2) medical history and risk factors; (3) clinical features at presentation, including symptoms (eg, typical chest discomfort) and signs (eg, blood pressure); and (4) infarct location (see online supplementary appendix 3 ).

Chest discomfort was defined as chest pain, chest tightness, chest pressure or other symptoms in the chest on record at admission that lasted $10 \mathrm{~min}$ or more. Other ischaemic symptoms included shortness of breath, pain at non-chest sites, nausea or fatigue on record at admission that lasted $10 \mathrm{~min}$ or more.

We did not consider some variables as candidates due to extremely low frequency $(<1 \%)$, including history of coronary artery bypass grafting (CABG), heart failure and peripheral vascular disease. Owing to high rates of missing in the medical records, we also excluded the following predictors: height $(86.6 \%$ missing $)$, weight (61.4\% missing) and Killip class (68.4\% missing). For age which was rarely unrecorded $(0.1 \%)$, we imputed the missing values as the overall median.

We transformed continuous variables, for example, age and systolic blood pressure (SBP), into categorical variables using a fractional polynomial approach with meaningful cut-off values, considering clinical and statistical associations with the mortality.

\section{Tool development}

We randomly divided the study sample into two subsets, with $75 \%$ in the derivation cohort, and $25 \%$ in the validation cohort. To assess the predictors of in-hospital mortality as, we developed a classification and regression tree (CART) analysis in the derivation cohort, ${ }^{24}$ which ensures better discrimination and less complexity in specifically identifying low-risk patients compared with conventional logistic regression. CART also allows for the assessment of the influence of covariates within partitions according to other covariates, which provides a more intuitive assessment of potential interactions. We considered all candidate predictors and used $\chi^{2}$ automatic interaction detection to divide the whole cohort into several groups with different predicted risk levels at each step by choosing the independent predictor that has the strongest interaction with the outcome, as well as the corresponding significant cut-off values. We set the minimum number of cases in each risk group as 100, to avoid overfitting of the model.

\section{Tool validation}

To test the ability of classification tree to identify patients with low risk of in-hospital mortality, we identified similar low-risk groups in the validation cohort using the classification rules established in the risk classification tree and compared the observed mortality with in the corresponding risk groups from the derivation cohort, with the OR and $95 \%$ CI. In the derivation and the validation cohorts, we evaluated discrimination of the classification tree with the c-statistic, which represents the area under the receiver-operating characteristic curves. ${ }^{25}$ We assessed the robustness of the classification tree using the c-statistics in the following patient subgroups: study year (2001, 2006 or 2011) and hospital capability for PCI (with or without). We also measured the performance of classification tree in ideal patients according to class I guideline recommendations who arrived to hospital within 12 hours with no contraindications. ${ }^{56}$

\section{Other statistical analyses}

For summary statistics, we presented percentages for categorical variables, and median and IQR for continuous variables. We performed $\chi^{2}$ tests for the bivariate associations and employed a stepwise (backward elimination) logistic regression to identify a minimal set of predictors independently associated with the mortality. To compare the difference in severity of disease across subgroups, we also employed Wilcoxon rank sum tests for mini-Global Registry of Acute Coronary Events (mini-GRACE) risk score, which is computed based on age, SBP on admission, heart rate, ST segment deviation, cardiac arrest and elevated cardiac biomarkers. ${ }^{26}$ All comparisons were 2-tailed, with a $\mathrm{p}<0.05$ considered statistically significant. All statistical analyses were performed with the SPSS 13.0 software package (SPSS, Chicago, USA).

\section{RESULTS}

\section{Characteristics of study cohort}

Among the 8059 fibrinolytic-eligible patients, the study cohort of 3741 patients who did not receive reperfusion therapy had a higher mini-GRACE risk score than those received the therapy $(146 \quad(126,165)$ versus 134 $(117,152), \mathrm{p}<0.001)$ and also than the patients transferred to other hospital or discharged alive within 24 hours $(140 \quad(120,160), \mathrm{p}<0.001)$ (figure 1) (see online supplementary appendix 4). In the study cohort, $34 \%$ were women, the median age was 69 years (IQR: 58-76). The median length of stay was 10 days (IQR: $6-$ 15). The overall in-hospital mortality was 14.7\%: 413 patients $(11.0 \%)$ died during the hospitalisation and $137(3.7 \%)$ withdrew from treatment due to terminal status. Among these patients, the median length of stay was 1 day (IQR 0-3) (figure 2). Nine independent predictors of in-hospital mortality, including advanced age, female gender, history of diabetes, chest discomfort, cardiac arrest at admission, low SBP at admission, heart rate $<50$ or $>100 \mathrm{bpm}$ at admission, and anterior, posterior or right ventricular infarction were retained in the final logistic model with the c-statistic of 0.77 (table 1).

\section{Predictors of mortality and classification tree}

Of the 19 predictors included in the CART analysis, the classification tree identified SBP at admission as the best 


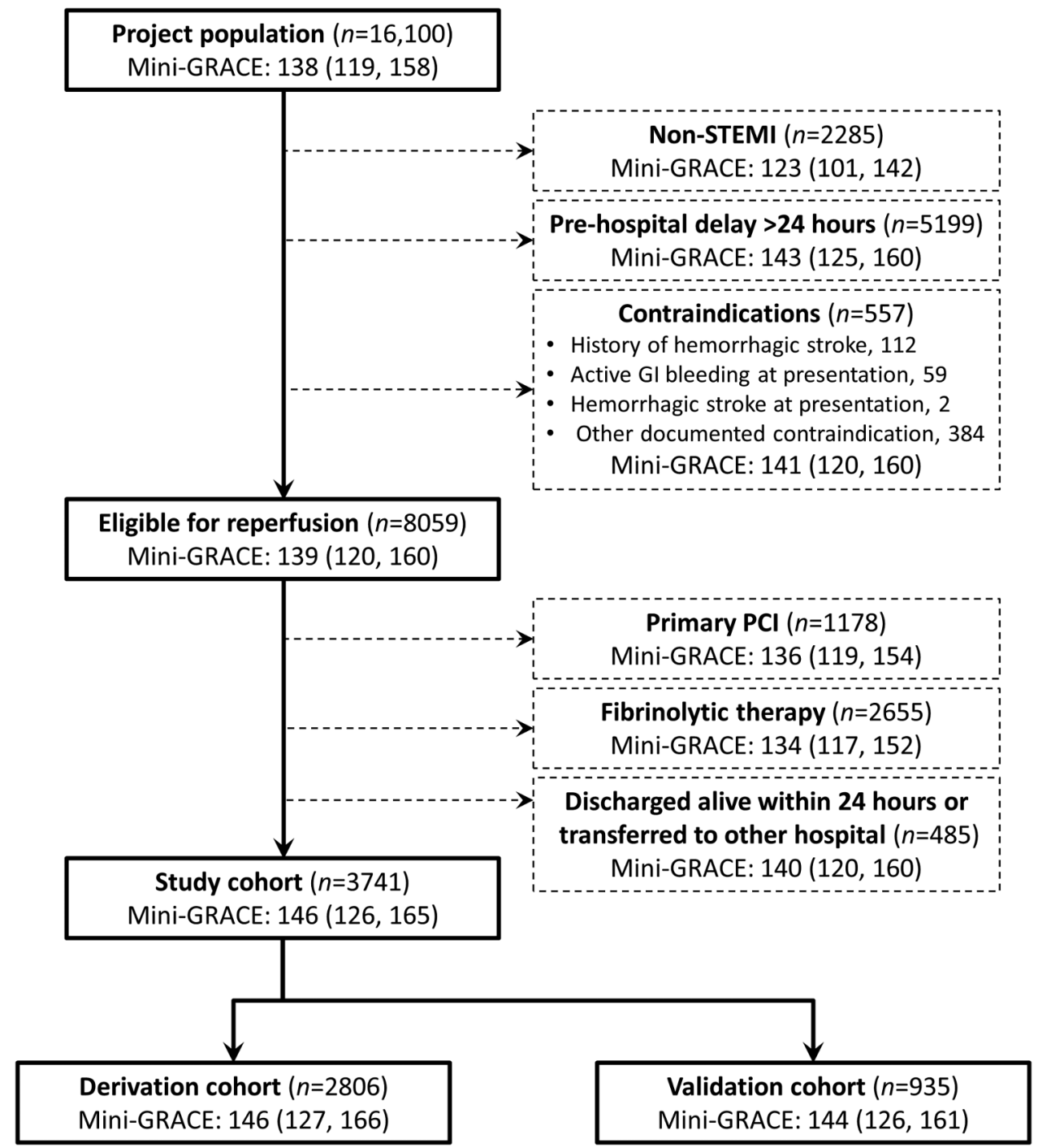

Figure 1 Flow chart: cohort for tool development and validation. To develop and validate the risk tool, we identified fibrinolytic-eligible patients, who had not received any reperfusion therapy. Eligibility was defined as patients with STEMI who arrived to the hospital within 24 hours of symptom onset and did not have contraindications to fibrinolytic therapy, including history of haemorrhagic stroke, active bleeding at presentation or any other physician-documented contraindication. We excluded the patients who were discharged alive within 24 hours or transferred to other hospitals. The mini-GRACE indicates the median and IQR of mini-GRACE risk score in each patients subgroup; GRACE, Global Registry of Acute Coronary Events; PCI, percutaneous coronary intervention; STEMI, ST segment elevation myocardial infarction.

independent discriminator for in-hospital mortality within the derivation cohort (figure 3). The average mortality risk was $11.3 \%$, in 2417 patients with a $\mathrm{SBP} \geq 100 \mathrm{~mm} \mathrm{Hg}$, who represented $86 \%$ of the derivation cohort. Within this subgroup, the next best predictor was the age-for 645 patients $<60$ years old $(23 \%$ of the derivation cohort), the observed risk was $3.6 \%$. Gender provided third highest additional predictive value, as these 528 men (19\% of derivation cohort) were less likely to suffer an in-hospital death. These three factors (SBP, age and gender) could be employed to identify one-fifth of the patients with an in-hospital mortality of $2.5 \%$. Furthermore, in this subgroup, 284 patients (10\% of the derivation cohort) with a non-anterior infarction had even lower risk for in-hospital mortality $(1.1 \%)$.
The derivation cohort was classified into 13 subgroups of different risk level in the CART analysis to create a classification tree. Chest discomfort and heart rate at admission also contributed in discrimination of other risk groups (see online supplementary appendix 5).

\section{Validation of the classification tree}

The classification tree had a $c$-statistic of 0.75 in the derivation cohort and 0.74 in the validation cohort, which indicates similarly good model discrimination. The model performed consistently well in risk discrimination in specific patient cohorts across different years and hospital with or without capability of PCI (figure 4). In 2489 ideal patients who were admitted within 12 hours after symptom onset, the classification tree, with a 


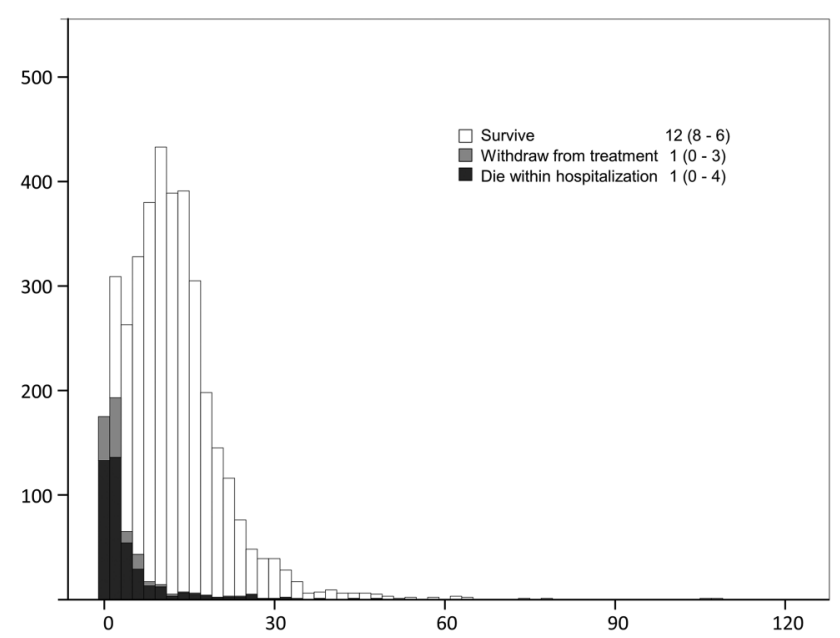

Figure 2 Length of stay (day) in patients with different outcomes. In a histogram, number of patients with different outcomes (vary in colours) were stacked within each 2-day interval of the length of stay. For patients died within hospitalisation, the length of stay was similar with those who withdrew from treatment due to a clinical terminal status, and much less than those survived.

$c$-statistic of 0.75 , also identified $499(20 \%)$ of them with an low average mortality risk of $3.0 \%$.

In the validation cohort, the average mortality risk was $3.9 \%$ in the 206 men under the age of 60 with an SBP at admission $\geq 100 \mathrm{~mm} \mathrm{Hg}$ (22\% of the cohort). Among these patients, 111 (12\% of the validation cohort) diagnosed as non-anterior AMI had a mortality risk of $2.7 \%$. The risk of these groups was similar with the counterparts in the derivation cohort ( $p$ value is 0.3 and 0.2 , respectively). The proportion of the low-risk group was slightly higher in PCI-capable hospitals (20\% vs $17 \%$, in non-PCI-capable hospitals, $\mathrm{p}=0.02$ ) and stays the same across different years $(\mathrm{p}=0.07)$.

\section{DISCUSSION}

In this nationally representative cohort of Chinese patients with STEMI who were eligible for but did not receive fibrinolytic therapy, we established an classification tree using three simple factors-SBP ( $\geq 100 \mathrm{~mm} \mathrm{Hg}$ ), age ( $<60$ years old) and gender (male), which can be ascertained easily and quickly at presentation, to identify a fifth of them with a baseline in-hospital mortality rate of $<3.0 \%$ in the absence of reperfusion. A half of these patients, with a non-anterior AMI, were at an even lower risk of $<1.5 \%$.

These findings challenge to conventional wisdom of a 'treat-everyone' approach to the fibrinolytic therapy for STEMI. Among patients with STEMI who are eligible for fibrinolytic therapy, guidelines uniformly support the therapy, a recommendation that is predicated on the assumption of a substantial absolute benefit for everyone, without respect to baseline mortality risk. However, presuming a generally consistent relative risk reduction across the spectrum of baseline risk, for low baseline risk patients with little to gain from the relative reduction in STEMI mortality risk, the overall benefit may be offset by the risk of major bleeding. For physicians to adequately balance individual benefit against potential risks, a simple tool that identifies low-risk patients, as presented here, has the potential to complement clinical judgement.

What have we known about the benefit and risk? The landmark trials suggest a relative reduction of $16 \%$ in 35 -day mortality by the fibrinolytic therapy (or about $18 \%$ excluding the risk of fatal bleeding complications), which seems slightly larger in younger patients. ${ }^{4}$ They likely present the best-case scenario with respect to bleeding because of the selected trial population. Moreover, these trials were typically conducted in an era before the widespread use of anticoagulants and dual antiplatelet therapy. ${ }^{4}$ More recent data from real-world registries suggest that the risk of bleeding with the treatment may be significantly greater than that suggested by the trials (as high as $1 \%$ for ICH and $5 \%$ for major bleeding). ${ }^{9-12} 27$ Notably, there are no landmark trials or registries involving data from China, where the incidence of haemorrhagic stroke is high, and the risk with fibrinolytic therapy may also be higher than in other countries. ${ }^{28}$

A question might be raised as to whether an in-hospital (average length of 10 days) mortality risk of $2.5 \%$ as we identified using the classification tree is sufficiently low to consider not treating a fibrinolytic-eligible patient. Using trials data, for every thousand low-risk patients not treated with fibrinolytic therapy, 25 of them would die during the hospitalisation with 35 additional deaths during days $0-35 .^{4}$ Assuming a relative risk reduction of $23 \%$ in the younger low-risk group regardless of fatal bleeding complications, 8 deaths would be avoided due to the treatment. This benefit is balanced by the risk of therapy. On the other hand, conservative estimates of the risk of ICH and non-intracranial bleeding would be $0.7 \%$ and $2 \%$, respectively. ${ }^{12}$ Thus, for every thousand treated, we expect to have 20 patients with non-intracranial major bleeding and 7 with ICH. Other literature would suggest that of those with ICH, four $(60 \%)$ would die and two $(25 \%)$ would be disabled. ${ }^{29}$ Moreover, since the group with low baseline risk were at the upper range of blood pressure, this group might have an even higher risk for ICH, which could further erode the potential benefit.

In resource-poor settings with limited timely access to PCI, fibrinolytic therapy is perhaps the most important strategy to reduce AMI mortality. In China, where there is an unprecedented rise in patients presenting to hospitals with AMI, efforts are underway to increase fibrinolytic use, especially in rural areas. In so doing, however, it is important to consider the balance of risks and benefits of fibrinolytic therapy to inform appropriate patient selection to optimise outcomes. Unfortunately, fibrinolytic-eligible patients with lower risk, for whom the benefit-risk ratio is less favourable, were even more likely to receive the treatment in the representative 
Table 1 Patients' characteristics and in-hospital mortality

\begin{tabular}{|c|c|c|c|c|c|c|c|}
\hline \multirow[b]{2}{*}{ Characteristics } & \multirow[b]{2}{*}{ Number } & \multirow{2}{*}{$\begin{array}{l}\text { Per } \\
\text { cent }\end{array}$} & \multirow{2}{*}{$\begin{array}{l}\text { In-hospital } \\
\text { mortality }\end{array}$} & \multicolumn{2}{|l|}{ Unadjusted } & \multicolumn{2}{|l|}{ Adjusted $†$} \\
\hline & & & & OR $(95 \% \mathrm{Cl})$ & p Value & OR $(95 \% \mathrm{Cl})$ & p Value \\
\hline Overall & 3741 & - & $550(14.7 \%)$ & - & - & - & - \\
\hline Age, year & & & & & $<0.001^{*}$ & & $<0.001$ \\
\hline$<60$ & 1006 & 26.9 & $74(7.4)$ & 1 & & 1 & \\
\hline $60-69$ & 899 & 24.0 & $108(12.0)$ & 1.72 (1.26 to 2.35$)$ & & 1.68 (1.20 to 2.36$)$ & \\
\hline $70-79$ & 1236 & 33.0 & $218(17.6)$ & 2.70 (2.04 to 3.56$)$ & & 2.49 (1.83 to 3.39$)$ & \\
\hline$\geq 80$ & 600 & 16.0 & $150(25.0)$ & 4.20 (3.11 to 5.67$)$ & & 3.72 (2.66 to 5.20$)$ & \\
\hline Female & 1281 & 34.2 & 264 (20.6) & 1.97 (1.64 to 2.37$)$ & $<0.001$ & 1.65 (1.34 to 2.02 ) & $<0.001$ \\
\hline History of CHD & & & & & 0.4 & & \\
\hline None & 2835 & 75.8 & $417(14.7)$ & 1 & & & \\
\hline Prior CHD, but no MI & 460 & 12.3 & 75 (16.3) & $1.13(0.86$ to 1.48$)$ & & & \\
\hline Prior MI & 446 & 11.9 & $58(13.0)$ & $0.87(0.65$ to 1.16$)$ & & & \\
\hline Prior PCl & 59 & 1.6 & $5(8.5)$ & $0.53(0.21$ to 1.34$)$ & 0.2 & & \\
\hline History of diabetes & 591 & 15.8 & $107(18.1)$ & 1.35 (1.07 to 1.70$)$ & 0.01 & 1.44 (1.12 to 1.87$)$ & 0.005 \\
\hline History of hypertension & 1756 & 46.9 & 239 (13.6) & 0.85 (0.71 to 1.02$)$ & 0.08 & & \\
\hline History of stroke & 450 & 12.0 & 78 (17.3) & 1.25 (0.96 to 1.63$)$ & 0.09 & & \\
\hline $\begin{array}{l}\text { History of chronic lung } \\
\text { disease }\end{array}$ & 186 & 5.0 & $29(15.6)$ & $1.08(0.72$ to 1.62$)$ & 0.7 & & \\
\hline Chest discomfort & 2654 & 70.9 & $334(12.6)$ & $0.58(0.48$ to 0.70$)$ & $<0.001$ & 0.80 (0.65 to 0.98$)$ & 0.03 \\
\hline $\begin{array}{l}\text { Other ischaemic } \\
\text { symptom }\end{array}$ & 1430 & 38.2 & $174(12.2)$ & $0.71(0.59$ to 0.86$)$ & 0.001 & & \\
\hline $\begin{array}{l}\text { Time from symptom to } \\
\text { admission, hour }\end{array}$ & & & & & $0.001^{*}$ & & \\
\hline$<3$ & 835 & 22.3 & $152(18.2)$ & 1 & & & \\
\hline $3-5$ & 781 & 20.9 & $113(14.5)$ & $0.76(0.58$ to 0.99$)$ & & & \\
\hline $6-11$ & 739 & 19.8 & $99(13.4)$ & $0.70(0.53$ to 0.92$)$ & & & \\
\hline $12-24$ & 1386 & 37.0 & $186(13.4)$ & $0.70(0.55$ to 0.88$)$ & & & \\
\hline $\begin{array}{l}\text { Cardiac arrest at } \\
\text { presentation }\end{array}$ & 51 & 1.4 & $20(39.2)$ & 3.85 (2.18 to 6.80$)$ & $<0.001$ & 2.51 (1.29 to 4.89$)$ & 0.007 \\
\hline $\begin{array}{l}\text { SBP at admission, } \\
\mathrm{mm} \mathrm{Hg}\end{array}$ & & & & & $<0.001^{*}$ & & $<0.001$ \\
\hline$<80$ & 142 & 3.8 & $89(62.7)$ & - & & 1 & \\
\hline 80-99 & 377 & 10.1 & $107(28.4)$ & $0.24(0.16$ to 0.35$)$ & & $0.26(0.17$ to 0.41$)$ & \\
\hline $100-119$ & 816 & 21.8 & $111(13.6)$ & 0.09 (0.06 to 0.14$)$ & & 0.11 (0.07 to 0.17$)$ & \\
\hline $120-139$ & 1063 & 28.4 & $120(11.2)$ & $0.08(0.05$ to 0.11$)$ & & 0.08 (0.05 to 0.12$)$ & \\
\hline$\geq 140$ & 1343 & 35.9 & $123(9.2)$ & $0.06(0.04$ to 0.09$)$ & & $0.06(0.04$ to 0.09$)$ & \\
\hline $\begin{array}{l}\text { Respiration rate at } \\
\text { admission > } 20 \text { bpm }\end{array}$ & 977 & 26.1 & $195(20.0)$ & 1.69 (1.40 to 2.05$)$ & $<0.001$ & & \\
\hline $\begin{array}{l}\text { Heart rate at admission, } \\
\text { bpm }\end{array}$ & & & & & $<0.001^{*}$ & & $<0.001$ \\
\hline$\leq 50$ & 245 & 6.5 & $56(22.9)$ & 2.31 (1.68 to 3.18$)$ & & 1.39 (0.95 to 2.01$)$ & \\
\hline $51-99$ & 2863 & 76.5 & $326(11.4)$ & 1 & & 1 & \\
\hline$\geq 100$ & 633 & 16.9 & $168(26.5)$ & 2.81 (2.28 to 3.47 ) & & 2.14 (1.70 to 2.71$)$ & \\
\hline \multicolumn{8}{|l|}{ Infarct location } \\
\hline Anterior $\ddagger$ & 1732 & 46.3 & $308(17.8)$ & 1.58 (1.32 to 1.90$)$ & $<0.001$ & 1.70 (1.38 to 2.11$)$ & $<0.001$ \\
\hline Inferior & 1395 & 37.3 & $195(14.0)$ & $0.91(0.75$ to 1.10$)$ & 0.3 & & \\
\hline $\begin{array}{l}\text { Posterior or right } \\
\text { ventricular }\end{array}$ & 466 & 12.5 & $81(17.4)$ & $1.26(0.97$ to 1.63$)$ & 0.08 & 1.46 (1.09 to 1.96$)$ & 0.01 \\
\hline $\begin{array}{l}\text { Left bundle branch } \\
\text { block }\end{array}$ & 70 & 1.9 & $19(27.1)$ & 2.20 (1.29 to 3.76$)$ & 0.004 & & \\
\hline
\end{tabular}

${ }^{*} p$ Value for trend.

†The c-statistics of the final logistic model is 0.77 .

flncluding anterior, extensive anterior, anteroseptal or lateral infarction.

$\mathrm{CHD}$, coronary heart disease; $\mathrm{MI}$, myocardial infarction; $\mathrm{PCl}$, percutaneous coronary intervention; $\mathrm{SBP}$, systolic blood pressure. 
Figure 3 The classification tree for decision-making in the derivation cohort. To identify the subgroup with lower risk of in-hospital mortality in the classification and regression tree (CART) analysis, systolic blood pressure $(\geq 100 \mathrm{~mm} \mathrm{Hg})$, age ( $<60$ years old), gender (male) and infarct location (non-anterior) was the best independent discriminator step by step. This flow chart demonstrates the size (proportion of the derivation cohort) and average risk in the lower-risk group at each step. SBP, systolic blood pressure; STEMI, ST segment elevation myocardial infarction.
2806 fibrinolytic therapy-eligible patients

Average mortality $=15.0 \%$

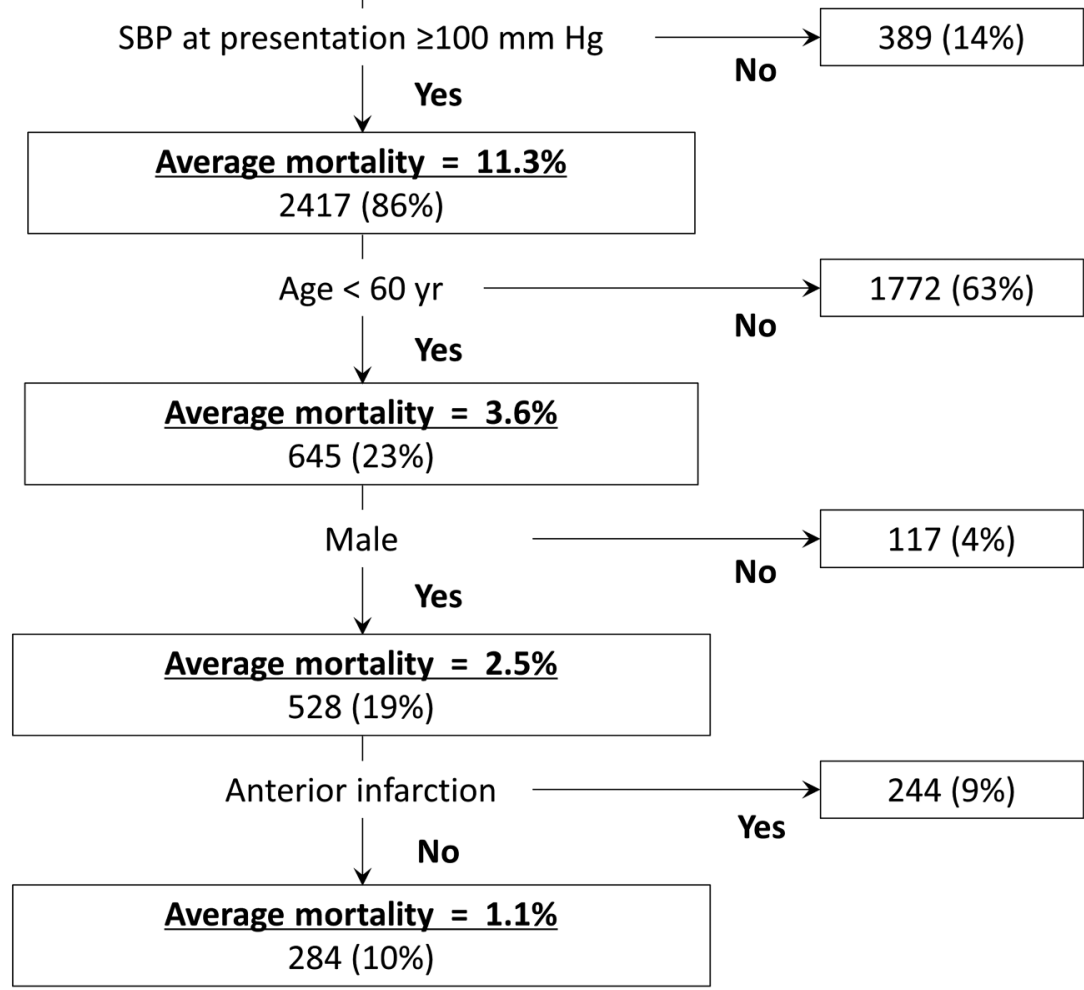

Yes miniature of the real-world practice, as the 'risktreatment paradox' suggested in many prior studies. ${ }^{30} 31$

The decision to use fibrinolytic therapy in patients presenting with STEMI requires the early identification of eligible patients, followed by a quick discussion with the patient about the risks and benefits of the therapy. Traditionally, decision-making almost always favoured treatment; however, our study shows that the baseline mortality risk may be sufficiently low in some patients to defer treatment, especially if there are reasons to believe that risk of bleeding is high. In these cases, a risk tool can assist physicians and patients in weighing the likelihood of benefit versus harm. This would also allow for individual interpretation of risk. For someone with a 3\% risk of dying (ie, a $97 \%$ probability of surviving), a chance to get 1 death prevented in 170 treated patients - the number needed to treat is 170 -may dominate their fear of the risk of a major bleeding; others may be more concerned with the consequences of bleeding and the potential for disability. Nevertheless, this tool is intended to inform practice, not dictate it. Moreover, there may always be some factors not in the model that indicate high risk, such as dynamic instability. Also, for some patients, the risk of bleeding might be so low as to make even a low possibility of benefit attractive.

Most contemporary risk stratification tools derived from reperfusion trials or registries population are not suitable for estimating the individual baseline risk, ${ }^{13-21}$ since a substantial proportion of patients within had received reperfusion therapy. In the Thrombolytic
Predictive Instrument, developed 20 years ago, a series of risk models specifically for either the mortality or ICH were integrated to balance the benefit with the risk of fibrinolytic therapy; ${ }^{27}$ however, the application in practice was limited, and there have been no efforts in the modern era to redefine the patient-level net benefit.

In addition, to strengthen the applicability, we created the tool considering the need for rapid assessment of patient risk in order to facilitate early decision-making even in less advanced facilities. Thus, we did not include those characteristics that were not universally available at

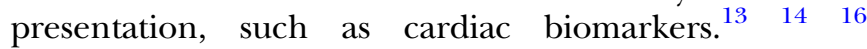
Moreover, because our risk tool was derived from an unselected population, representing an entire range of patients experiencing STEMI encountered in 'realworld' practice, in hospitals with widely varying resource capacities, it is widely relevant to China's diverse healthcare systems. The validity of this tool in other lowincome and middle-income, resource-poor countries needs to be tested.

To identify a low-risk subgroup of eligible patients, we conservatively considered treatment withdrawal due to terminal clinical status as a component of the primary endpoint. While this approach may overestimate the risk, our findings indicate that a low-risk group can be reliably identified, even using a broad definition of in-hospital mortality. Moreover, considering many patients in the study cohort were not being treated optimally by other effective therapies, ${ }^{22}$ the 'true' mortality risk in the low-risk group would be expected to be even lower. 


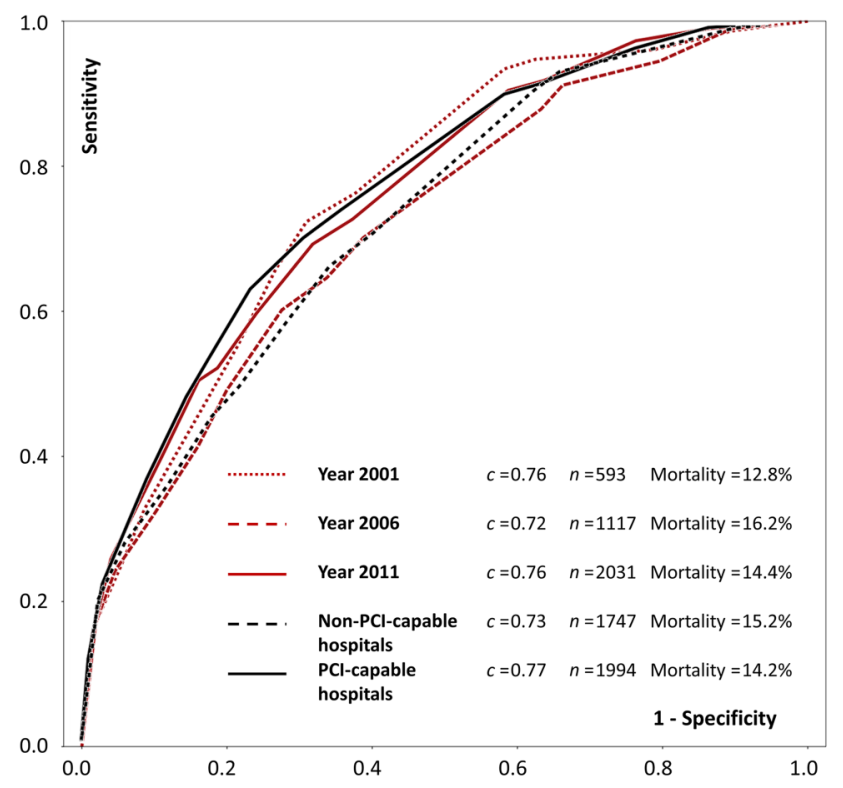

Figure 4 Validation of classification tree in different subgroups: the receiver-operating characteristic curve and the c-statistics. $\mathrm{PCl}$, percutaneous coronary intervention.

\section{Limitations}

Certain factors should be considered in the interpretation of our study. First, the decision analysis involved only the short-term benefits since we were unable to assess long-term outcomes. Nevertheless, it should be noted that in long-term follow-up of prior trials on fibrinolytic therapy, the entire benefit of the drug was concentrated in the short-term period. ${ }^{32}$ Second, data about short-term death after the discharge were unavailable in the medical records. However, since the length of stay in our cohort was relatively longer than in western countries (10 vs 7 days), ${ }^{33}$ most of the short-term outcomes would have likely occurred within the hospitalisation. We also used a composite endpoint including withdrawal from treatment due to a terminal status at discharge, which was a conservative assumption, given that we are seeking to develop an approach to identify low-risk patients. Third, patient eligibility and data collection on some predictors were based on local diagnosis in medical records, which may cause concern on external and internal validity of the findings. Nevertheless, the effects should be small as in a central auditing based on the first ECG of 300 randomly selected medical records; the accuracy of local diagnosis on AMI types was acceptable with a concordance of $94.7 \%$. Fourth, the estimation of absolute benefit of fibrinolytic therapy was based on the assumption that the relative benefit is not significantly heterogeneous across subgroups of genders and SBP, as the landmark trials suggested. However, we lack the power to detect the realistic amount of benefit in low-risk group empirically, even the overall sample size was relatively large. Fifth, in this retrospective study based on chart review, important predictors like Killip class and amount of ST segment elevation had to be excluded due to missing in the medical records. Also, it is possible that some of the patients included had undocumented contraindications to therapy, but that would only make our finding more conservative by elevating the baseline risk since ineligible patients tend to be in worse condition. And finally, the tool needs prospective validation, to make sure of a prospective application.

\section{CONCLUSION}

The current study and tools helped identify a substantial subgroup of eligible patients, among whom the net benefit of fibrinolytic therapy is likely marginal, considering major bleeding complications and other realistic factors. A quantitative estimation of the potential risks and benefits may facilitate more informed, individualised decision-making, which reminded us to consider baseline risk as an important criterion in the balancing. As fibrinolytic therapy is the dominant reperfusion strategy in developing countries, its use needs to be conducted in a careful and personalised way, in order to achieve its maximum capacity in saving lives.

Author affiliations

${ }^{1}$ State Key Laboratory of Cardiovascular Disease, National Clinical Research Center for Cardiovascular Diseases, Fuwai Hospital, National Center for Cardiovascular Diseases, Chinese Academy of Medical Sciences and Peking Union Medical College, Beijing, China

${ }^{2}$ Division of Cardiology, University of Colorado Anschutz Medical Campus, Aurora, Colorado, USA

${ }^{3}$ Saint Luke's Mid America Heart Institute/University of Missouri-Kansas City, Kansas City, Missouri, USA

${ }^{4}$ Center for Outcomes Research and Evaluation, Yale-New Haven Hospital, New Haven, Connecticut, USA

${ }^{5}$ Section of Cardiovascular Medicine, Department of Internal Medicine, Yale School of Medicine; Center for Outcomes Research and Evaluation, Yale-New Haven Hospital; Robert Wood Johnson Foundation Clinical Scholars Program, Department of Medicine, Yale School of Medicine; Department of Health Policy and Management, Yale School of Public Health, New Haven, Connecticut, USA

Acknowledgements The authors appreciate the multiple contributions made by study teams at the China Oxford Centre for International Health Research, the Yale-New Haven Hospital Center for Outcomes Research and Evaluation and all the study sites in the realms of study design and operations (see online supplementary appendix 6 and 7), particularly the data collection by $Y_{i}$ $\mathrm{Pi}$, Jiamin Liu, Wuhanbilige Hundei, Haibo Zhang, Lihua Zhang, Xue Du, Wenchi Guan, Xin Zheng, and Yuanlin Guo. They appreciate the advice of analysis by Haiqun Lin. They also appreciate the editing by Sudhakar Nuti and Brian Wayda. They are grateful for the support provided by the Chinese government.

Contributors HMK and LJ conceived the China PEACE study and take responsibility for all aspects of it. XL, JL, FAM, JAS, ZL, HMK and LJ designed the study. $X L$ wrote the first draft of the article, with further contributions from JL, FAM, JAS, ZL, HMK and LJ. XL did statistical analysis, with support from ZL. All authors interpreted data and approved the final version of the article.

Funding This project was partly supported by the Research Special Fund for Public Welfare Industry of Health (201202025) from the National Health and Family Planning Commission of China, and the National Key Technology R\&D Program (2013BAI09B01) from the Ministry of Science and Technology of China. HMK is supported by grant U01 HL105270-05 (Center for Cardiovascular Outcomes Research at Yale University) from the National Heart, Lung, and Blood Institute. 
Disclaimer The views and opinions expressed in this article are those of the authors and do not reflect an official position of the institution or funder.

Competing interests HMK reports research grants from Medtronic and Johnson \& Johnson, through Yale University, to develop methods of clinical trial data sharing, and the chair of a cardiac scientific advisory board for UnitedHealth.

Ethics approval Fuwai Hospital, National Center for Cardiovascular Diseases of China.

\section{Provenance and peer review Not commissioned; externally peer reviewed.}

Data sharing statement In addition to data in this manuscript, all data in the China PEACE-Retrospective AMI Study, including over 900 variables of 16100 patients, are available to investigators from 162 local sites now and to the whole research community in 2 years. Subdata set of each local site is stored in its own workstation. The national overall data sets are stored in servers of Fuwai Hospital. Local investigators can submit proposal for overall data use for research purposes.

Open Access This is an Open Access article distributed in accordance with the Creative Commons Attribution Non Commercial (CC BY-NC 4.0) license, which permits others to distribute, remix, adapt, build upon this work noncommercially, and license their derivative works on different terms, provided the original work is properly cited and the use is non-commercial. See: http:// creativecommons.org/licenses/by-nc/4.0/

\section{REFERENCES}

1. Chen $Y$, Jiang $L$, Zhang $Q$, et al. Doctor-reported hospital management of acute coronary syndrome in China: a nationwide survey of 1029 hospitals in 30 provinces. World J Cardiovasc Dis 2012;2:168-76.

2. Boden WE, Eagle K, Granger CB. Reperfusion strategies in acute ST-segment elevation myocardial infarctionA comprehensive review of contemporary management options. J Am Coll Cardiol 2007:50:917-29.

3. Eagle KA, Goodman SG, Avezum A, et al. Practice variation and missed opportunities for reperfusion in ST-segment-elevation myocardial infarction: findings from the Global Registry of Acute Coronary Events (GRACE). Lancet 2002;359:373-7.

4. Fibrinolytic Therapy Trialists' (FTT) Collaborative Group. Indications for fibrinolytic therapy in suspected acute myocardial infarction: collaborative overview of early mortality and major morbidity results from all randomised trials of more than 1000 patients. Lancet 1994:343:311-22

5. O'Gara PT, Kushner FG, Ascheim DD, et al., American College of Emergency Physicians, Society for Cardiovascular Angiography and Interventions. 2013 ACCF/AHA guideline for the management of ST-elevation myocardial infarction: a report of the American College of Cardiology Foundation/American Heart Association Task Force on Practice Guidelines. J Am Coll Cardiol 2013;61:e78-e140.

6. Steg PG, James SK, Atar D, et al., Task Force on the management of ST-segment elevation acute myocardial infarction of the European Society of Cardiology (ESC). ESC Guidelines for the management of acute myocardial infarction in patients presenting with ST-segment elevation. Eur Heart J 2012;33:2569-619.

7. Antman EM, Anbe DT, Armstrong PW, et al., American College of Cardiology, American Heart Association Task Force on Practice Guidelines, Canadian Cardiovascular Society. ACC/AHA guidelines for the management of patients with ST-elevation myocardial infarction: a report of the American College of Cardiology/American Heart Association Task Force on Practice Guidelines (Committee to Revise the 1999 Guidelines for the Management of Patients With Acute Myocardial Infarction). Circulation 2004;110:e82-e292.

8. Van de Werf F, Ardissino D, Betriu A, et al. Management of acute myocardial infarction in patients presenting with ST-segment elevation: the Task Force on the Management of Acute Myocardial Infarction of the European Society of Cardiology. Eur Heart $J$ 2003;24:28-66.

9. Moscucci M, Fox KA, Cannon CP, et al. Predictors of major bleeding in acute coronary syndromes: the Global Registry of Acute Coronary Events (GRACE). Eur Heart J 2003;24:1815-23.

10. Huynh T, Cox JL, Massel D, et al. Predictors of intracranial hemorrhage with fibrinolytic therapy in unselected community patients: a report from the FASTRAK II project. Am Heart J 2004;148:86-91.

11. Gurwitz JH, Gore JM, Goldberg RJ, et al. Risk for intracranial hemorrhage after tissue plasminogen activator treatment for acute myocardial infarction. Ann Intern Med 1998;129:597-604.
12. Brass LM, Lichtman JH, Wang $\mathrm{Y}$, et al. Intracranial hemorrhage associated with thrombolytic therapy for elderly patients with acute myocardial infarction: results from the Cooperative Cardiovascular Project. Stroke 2000;31:1802-11.

13. Granger CB, Goldberg RJ, Dabbous O, et al. Predictors of hospital mortality in the global registry of acute coronary events. Arch Intern Med 2003;163:2345-53.

14. Antman EM, Cohen M, Bernink PJ, et al. The TIMI risk score for unstable angina/non-ST elevation MI. JAMA 2000;284:835-42.

15. Morrow DA, Antman EM, Charlesworth A, et al. TIMI risk score for ST-elevation myocardial infarction: a convenient, bedside, clinical score for risk assessment at presentation an intravenous nPA for treatment of infarcting myocardium early II trial substudy. Circulation 2000;102:2031-7.

16. Chin CT, Chen AY, Wang TY, et al. Risk adjustment for in-hospital mortality of contemporary patients with acute myocardial infarction: the acute coronary treatment and intervention outcomes network (ACTION) registry-get with the guidelines (GWTG) acute myocardial infarction mortality model and risk score. Am Heart $J$ 2011;161:113-122.e2.

17. Morrow DA, Antman EM, Giugliano RP, et al. A simple risk index for rapid initial triage of patients with ST-elevation myocardial infarction: an InTIME II substudy. Lancet 2001;358:1571-5.

18. Lee KL, Woodlief LH, Topol EJ, et al. Predictors of 30-day mortality in the era of reperfusion for acute myocardial infarction results from an international trial of 41021 patients. Circulation 1995;91:1659-68.

19. Normand ST, Glickman ME, Sharma RG, et al. Using admission characteristics to predict short-term mortality from myocardial infarction in elderly patients. Results from the Cooperative Cardiovascular Project. JAMA 1996;275:1322-8.

20. Krumholz HM, Chen J, Wang Y, et al. Comparing AMI mortality among hospitals in patients 65 years of age and older evaluating methods of risk adjustment. Circulation 1999;99:2986-92.

21. Krumholz HM, Wang Y, Mattera JA, et al. An administrative claims model suitable for profiling hospital performance based on 30-day mortality rates among patients with an acute myocardial infarction. Circulation 2006;113:1683-92.

22. Li J, Li X, Wang $Q$, et al. ST-segment elevation myocardial infarction in China from 2001 to 2011 (the China PEACE-Retrospective Acute Myocardial Infarction Study): a retrospective analysis of hospital data. Lancet. 2015;385:441-51.

23. Dharmarajan K, Li J, Li X, et al. The China Patient-centered Evaluative Assessment of Cardiac Events (China PEACE) retrospective study of acute myocardial infarction: study design. Circ Cardiovasc Qual Outcomes 2013;6:732-40.

24. Steadman HJ, Silver E, Monahan J, et al. A classification tree approach to the development of actuarial violence risk assessment tools. Law Hum Behav 2000;24:83-100.

25. Hanley JA, McNeil BJ. The meaning and use of the area under a receiver operating characteristic (ROC) curve. Radiology 1982;143:29-36.

26. Simms AD, Reynolds S, Pieper K, et al. Evaluation of the NICE mini-GRACE risk scores for acute myocardial infarction using the Myocardial Ischaemia National Audit Project (MINAP) 2003-2009: National Institute for Cardiovascular Outcomes Research (NICOR). Heart 2013;99:35-40.

27. Selker HP, Griffith JL, Beshansky JR, et al. Patient-specific predictions of outcomes in myocardial infarction for real-time emergency use: a thrombolytic predictive instrument. Ann Intern Med 1997;127:538-56.

28. Jiang B, Wang WZ, Chen $\mathrm{H}$, et al. Incidence and trends of stroke and its subtypes in China results from three large cities. Stroke 2006;37:63-5

29. Gore JM, Granger CB, Simoons ML, et al. Stroke after thrombolysis mortality and functional outcomes in the GUSTO-I trial. Circulation 1995;92:2811-18.

30. Elbarouni B, Goodman SG, Yan RT, et al. Validation of the Global Registry of Acute Coronary Event (GRACE) risk score for in-hospital mortality in patients with acute coronary syndrome in Canada. Am Heart J 2009;158:392-9.

31. Yan AT, Yan RT, Tan M, et al. Management patterns in relation to risk stratification among patients with non-ST elevation acute coronary syndromes. Arch Intern Med 2007;167:1009-16.

32. Baigent $\mathrm{C}$, Collins $\mathrm{R}$, Appleby $\mathrm{P}$, et al. ISIS-2: 10 year survival among patients with suspected acute myocardial infarction in randomised comparison of intravenous streptokinase, oral aspirin, both, or neither. BMJ 1998;316:1337-43.

33. Drye EE, Normand SL, Wang Y, et al. Comparison of hospital risk-standardized mortality rates calculated by using in-hospital and 30-day models: an observational study with implications for hospital profiling. Ann Intern Med 2012;156:19-26. 